\title{
Equivalent-Circuit Interconnect Modeling Based on The Fifth-Order Differential Quadrature Methods
}

\author{
Qinwei Xu, Student Member, IEEE and Pinaki Mazumder, Fellow, IEEE
}

\begin{abstract}
This paper introduces an efficient and passive discrete modeling technique for estimating signal propagation delays through on-chip long interconnects that are represented as distributed $R L C$ transmission lines. The proposed delay model is based on a less frequently used numerical approximation technique, called the differential quadrature method (DQM). The DQM can compute the partial derivative of a function at any arbitrary point located within a prespecified closed domain of the function by quickly estimating the weighted linear sum of values of the function at a relatively small set of well-chosen grid points within the domain. By using the fifth-order DQM, a new approximation framework is constructed in this paper for discretizing the distributed $R L C$ interconnect and thereafter modeling its delay. Due to high efficiency of DQM approximation, the proposed framework requires only few grid points to achieve good accuracy. The presented equivalent-circuit model appears like the ones derived by the finite difference (FD) method. However, it has higher accuracy and less internal nodes than generated by the FD-based modeling. The fifth-order DQM modeling technique is shown to preserve passivity. It has linear forms that are compatible with the passive order-reduction algorithm for linear network. Numerical experiments show that the proposed modeling approach leads to high accuracy as well as high efficiency.
\end{abstract}

Index Terms-Differential quadrature method (DQM), discrete transmission line model, equivalent circuit, interconnect modeling, passivity, transient simulation.

\section{INTRODUCTION}

W ITH the feature size of VLSI technology continually shrinking and chip area simultaneously increasing, the VLSI circuit simulators are now encountering formidable challenges to precisely model various passive circuit elements such as multilayer interconnects, substrates, wells, packaging structures, etc., so that their electrical characteristics can be presented to circuit simulator along with short-channel device models. As the length of on-chip global interconnects increases to few centimeters, at very high-speed of operation, when the time of flight of signals is comparable to their rise/fall times, the parasitic inductance of passive elements plays a dominant role in determining the signal waveforms and propagation delay. In order to accurately estimate the signal integrity and circuit speed, the interconnect modeling algorithms that are now being used in circuit simulators must model these interconnects as distributed $R L C$ transmission lines.

Manuscript received December 3, 2002. This work was supported in part by MURI Grant and in part by an ONR Grant.

The authors are with the Department of Electrical Engineering and Computer Science, University of Michigan, Ann Arbor, MI 48109 USA (e-mail: qwxu@eecs.umich.edu; mazum@eecs.umich.edu).

Digital Object Identifier 10.1109/TVLSI.2003.817522
Over the past decades, many methods were developed in order to model the propagation delays through transmission lines. Transmission line characteristics are usually represented by transcendental functions where signal frequency and line length are arguments. As transmission lines are generally characterized in the frequency domain and are usually terminated with nonlinear loads, the time domain models of transmission lines are derived by applying the convolution operation. This allows us to observe the transient response of the transmission line [1]. Considerable amount of research has been done in the literature to convert the frequency-domain solutions to time-domain responses. The typical methods are the fast Fourier transform (FFT) and numerical inverse Laplace transform (NILT), which can be employed in most cases. When the inverse transforms are directly used to find the time models, the computation complexity becomes proportional to the square of the simulation time, thus significantly slowing down the circuit simulators. Moreover, these methods have an intrinsic shortcoming due to aliasing error that introduces inaccuracy to evaluate the delay. On the contrary, the method of characteristics (MC) can accurately separate the time delay due to distributed inductance and capacitance. It is also efficient and accurate for solving the telegrapher's equations of transmission lines. Transmission line models obtained by MC can be implemented into the existing circuit simulators such as SPICE. However, the frequency domain equations of $\mathrm{MC}$ are not linear; therefore, they cannot be incorporated in model-order reduction.

Recently the reduced-order macromodeling has been widely used. Asymptotic waveform evaluation (AWE) technique was the first well-known macro-modeling method for representing the general linear networks [2], [3]. However, AWE macromodeling technique had two intrinsic limitations, namely poor accuracy and instability. These shortcomings have been overcome in subsequent research work that led to the development of the complex frequency hopping (CFH) method [4], [5]. Unlike AWE that uses a single expansion point, $\mathrm{CFH}$ performs the Taylor series expansion of the characteristic function at multiple points on the imaginary axis, which are known as hopping points. Frequency hopping on the complex plane allows the CFH to improve its accuracy and the process is inherently stable since it has no poles on the right plane. The residues of the poles are determined using a selected set of low-order moments generated at various expansion points for frequency hopping. CFH preserves the poles of the transfer function rather than the moments, and it circumvents the ill-conditions encountered in AWE. However, each hop of $\mathrm{CFH}$ requires its own expensive processing time and complex 
mathematical manipulations while AWE only requires one expansion. Two other techniques employing two-pole [6] or multipoint moment matching methods [7] can also generate reduced-order macromodels.

Currently the development of model reduction algorithms is in progress for macromodeling very large linear network containing distributed transmission lines. The realistic integrated systems generally include a large number of state variables associated with the distributed interconnections and lumped $R L C$ elements. In order to overcome this difficulty, model reduction has been employed. A large system is at first partitioned into both nonlinear and linear systems; then the algorithms of model-order reduction are performed to linear parts only. It is very important that the reduced-order model maintain the passivity properties of the original circuit. Though the Krylov subspace techniques such as Padé Via Lancoz (PVL) [8] and Matrix Padé Via Lanczos (MPVL) [9] are passive in some cases, the Padé-based reduced-order model cannot guarantee passivity for general $R L C$ circuit. On the other hand, a new direction for passive reduced-order model shown in [10], is based on congruence transformations. An extended technique based on Arnoldi's method with congruence transformations is presented in the literature [11], in which the PRIMA algorithm was demonstrated as a milestone to develop passive reduced-order models.

Although the algorithms of model reduction are well developed and are being continuously improved, it can only handle the finite-order systems in the forms of state equations. Specifically, the original system to be reduced should be described in the form of $A+s B=C$. Transmission lines, however, are represented by nonlinear partial differential equations, which are infinite order systems. Therefore, it becomes imperative to discretize the transmission lines into models involving finite state variables so that they are stamped into the stencil of $A+s B=C$ prior to the reduction process. However, the finite-order transmission-line models must preserve the passivity of the original distributed transmission lines.

One of the discretization methods is the distributed modeling, which implicitly obtains state equations with finite state variables [7]. On the other hand, the most effort to develop finite-order models of distributed transmission line is focused on direct discretization approaches, which generally select grid points along the lines. As partial differential equations have been long approximately solved by finite difference (FD) or finite element (FE) methods [12], the discretization of transmission lines is far from a new topic. For instance, it is the FD discrete model that represents the distributed transmission lines in the linear network handled by the original PRIMA algorithm [11]. A low-order finite method to model transmission lines gives well understandable physical explanation of lumped element equivalent circuits. Such a popular approach for discrete modeling directly segments the line into sections whose lengths are chosen to be small fractions of the minimum wavelength. Despite its simplicity, it has the disadvantage that the number of grid points, depending on the minimum wavelength, is generally as large as dozens. Consequently, such an approach results in very large numbers of lumped elements for accurate modeling and thus sharply increases the number of state variables of the whole circuit. A compact difference method is employed in literature [13], which has fourth-order accuracy. In this discretization approach, the number of unknowns per wavelength required for highly accurate modeling is smaller and its dependence on the electrical length of the line is weaker.

The drawback of low-order finite methods can be removed by using the high-order finite methods or pseudospectral methods [14]. The mathematical fundamental of FD schemes is the Taylor series expansion. The scheme of low-order finite method is determined by low-order Taylor series, while the scheme of high-order finite method is determined by high-order Taylor series. In general, the high-order schemes have a high-order truncation error. Thus, to achieve the required accuracy, the mesh size used by the high-order schemes can be much less than that used by low-order schemes. As a result, the high-order schemes can obtain accurate numerical solutions using very few mesh points. Chebyshev polynomial representation, a kind of pseudospectral methods, has been used to model transmission lines and shown high efficiency [15]. However, it cannot guarantee passivity.

In this paper, the fifth-order differential quadrature method (DQM) is employed for passive modeling of transmission lines. A numerical technique similar to the spectral method, DQM was originally developed by mathematicians to approximately solve nonlinear partial differential equations (PDE) [16]. As an alternative to the FD and FE methods, the DQM gained use in solving differential equations in many engineering areas. The idea of DQMs is to quickly compute the derivative of a function at any grid point within its bounded domain by estimating a weighted linear sum of values of the function at a small set of points belonging to the domain. Recently general DQMs have been employed for interconnect modeling [17], and high efficiency is observed. However, the passivity cannot be guaranteed. This paper adapts the DQM for passive interconnect modeling in the following steps.

At first, the fifth-order DQM is investigated and the specific approximation frame is derived for the modeling of transmission lines. Then following the conventional FD model of transmission lines, the discrete models are obtained by using the fifth-order DQM-based approximation framework. Like FD-based models, the proposed discrete models can be incorporated into popular all-purpose simulators. Due to the super efficiency of DQM, the proposed discrete modeling approaches give high-approximation accuracy using moderately few grid points, which improves the computational efficiency of transmission line modeling. The generated discrete models are theoretically proved to be passive, and have the linear form of $A+B s=C$, therefore, they are compatible to the reduced order algorithm for linear circuit reduction. However, this presentation focuses on the DQM-based discrete modeling, and will not be concerned on model-order-reduction techiques.

The organization of this paper is as follows. In Section II, the mechanisms of two kinds of DQMs are reviewed and the approximation framework is derived by using fifth-order DQMs. The fifth-order DQM-based model of single transmission line are discussed in Section III and it is extended for multiconductor transmission lines (MTLs) in Section IV. In Section V the accuracy and efficiency rules of fifth-order DQM-based mod- 
eling approaches are investigated. Numerical examples are presented and the results of the proposed approaches are compared with the results obtained using other methods in the following sections.

\section{APPROXIMATION FRAMEWORK FOR TRANSMISSION LINES}

The presented approximation frame is based on DQM, whose mathematical basis is the interpolation function. In the practical application of distributed transmission lines, the $s$-domain distributed voltages or currents $u=u(x, s)=\{V(x, s), I(x, s)\}$ can be approximated by means of interpolation as follows:

$$
u(x, s) \simeq u^{(N)}(x, s)=\sum_{i=1}^{N} L_{i}(x) u_{i}(s) x \in[0,1]
$$

where $u_{i}(s)=\left\{V\left(x_{i}, s\right), I\left(x_{i}, s\right)\right\}$ and $L_{i}(x)$ is an interpolation function determined by $x_{i}$ 's and interpolating formulation.

\section{A. Differential Quadrature Methods}

We employ the DQM to approximate the first-order derivative of the distributed voltages or currents along transmission lines [16], given by

$$
\frac{\partial}{\partial x} u\left(x_{i}, s\right)=\sum_{j=1}^{N} a_{i j} u_{j}(s)
$$

where $x \in[0,1], 0=x_{1}<x_{2}<\cdots<x_{N}=1$, and $i, j=$ $1,2, \ldots, N$. Equation (2) is called the $N$ th-order differential quadrature approximation.

The key procedure to this technique is to determine the differential quadrature (DQ) coefficients $a_{i j}$. Once the locations of grid points are selected, the DQ coefficients are uniquely determined by the formation of interpolation $L_{i}(x)$. Following the concept of the weighting residual method, one way suggested by Bellman etc. in [16] is to let (2) be exact for test functions

$$
g(x)=\left\{1, x, x^{2}, \ldots, x^{N-1}\right\} .
$$

By substituting every item in the function set into (2), a set of equations having Vandermonde matrix is obtained, and the coefficients $a_{i j}$ can be calculated by solving the equations. Further studies give the coefficients by the following closed-form formulas:

$$
\begin{aligned}
a_{i j} & =\frac{1}{\left(x_{i}-x_{j}\right)} \frac{\prod_{k \neq j}\left(x_{j}-x_{k}\right)}{\prod_{k \neq i}\left(x_{i}-x_{k}\right)}, i \neq j \\
a_{i i} & =\sum_{i \neq j} \frac{1}{x_{i}-x_{j}} .
\end{aligned}
$$

Such an approach is called polynomial-based differential quadrature (PDQ). The above process shows that the PDQ method is closely related to the collocation or pseudospectral method [14]. Its principal advantages over the latter, however, lies in its simplicity of using grid spacing without any restriction.

Another way to determine DQ coefficients is to employ harmonic (triangular) functions, called harmonic differential quadrature (HDQ). An $N$ th-order Fourier expansion is a linear

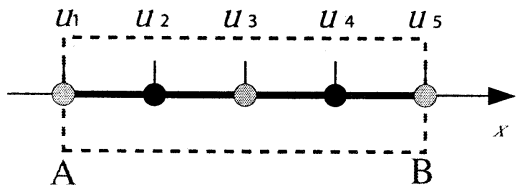

Fig. 1. Approximation framework based on fifth-order DQM.

combination in an $N$-dimension linear subspace, which is spanned by the following orthogonal base:

$$
g(x)=\left\{1, \sin \pi x, \cos \pi x, \ldots, \sin \frac{N-1}{2} \pi x, \cos \frac{N-1}{2} \pi x\right\}
$$

where $N$ is the number of grid points that is normally an odd number. In order to determine the weighting coefficients using sine and cosine functions, let (2) be exact when test functions take the set of (5), then the weighting coefficients are determined by

$$
\sum_{j=1}^{N} a_{i j} g\left(x_{j}\right)=\frac{d}{d x} g\left(x_{i}\right), \quad i=1,2, \ldots, N .
$$

The explicit formulas in this case are given by [18]

$$
\begin{aligned}
a_{i j} & =\frac{\frac{\pi}{2}}{\sin \left(\left(x_{i}-x_{j}\right) \frac{\pi}{2}\right)} \frac{\prod_{k \neq j} \sin \left(\left(x_{j}-x_{k}\right) \frac{\pi}{2}\right)}{\prod_{k \neq i} \sin \left(\left(x_{i}-x_{k}\right) \frac{\pi}{2}\right)}, i \neq j \\
a_{i i} & =-\sum_{k \neq i} a_{i k} .
\end{aligned}
$$

All the DQM coefficients in (4) [or (6)] form an $N \times N$ matrix, called DQM operator. For the application below, here we give a property of DQM operators.

Theorem 1: If the grid points are equally spacing, then the DQM operator is inverse symmetric with respect to the central point of the matrix, i.e.,

$$
a_{i j}=-a_{(N-i+1)(N-j+1)} .
$$

Proof: Equation (7) can prove to be true by using either (4) or (6).

\section{B. Approximation Framework Based on Fifth-Order DQM}

Next we use fifth-order DQMs to construct the approximations of the distributed voltages (or currents) along transmission lines. For simplicity and without loss of generality, we study the fifth-order DQM applied to a single transmission line at first. Assuming that $A B$ is a sliding window containing a section of the transmission line, if it is uniformly segmented into four subsections, then five grid points are obtained as shown in Fig. 1.

Normalizing the section AB into a unit length, the DQM approximation in this case is represented by

$$
\left[\begin{array}{c}
u_{1}^{\prime} \\
u_{2}^{\prime} \\
\vdots \\
u_{5}^{\prime}
\end{array}\right]=\left[\begin{array}{cccc}
a_{11} & a_{12} & \ldots & a_{15} \\
a_{21} & a_{22} & \ldots & a_{25} \\
\vdots & \vdots & \ddots & \vdots \\
a_{51} & a_{52} & \ldots & a_{55}
\end{array}\right]\left[\begin{array}{c}
u_{1} \\
u_{2} \\
\vdots \\
u_{5}
\end{array}\right]
$$

where $u_{i}=u\left(x_{i}, s\right)$ is the $s$-domain voltage at point $x_{i}, u_{i}^{\prime} \mathrm{s}$ denote the derivatives

$$
u_{i}^{\prime}=\left.\frac{d}{d x} u(x, s)\right|_{x=x_{i}}
$$




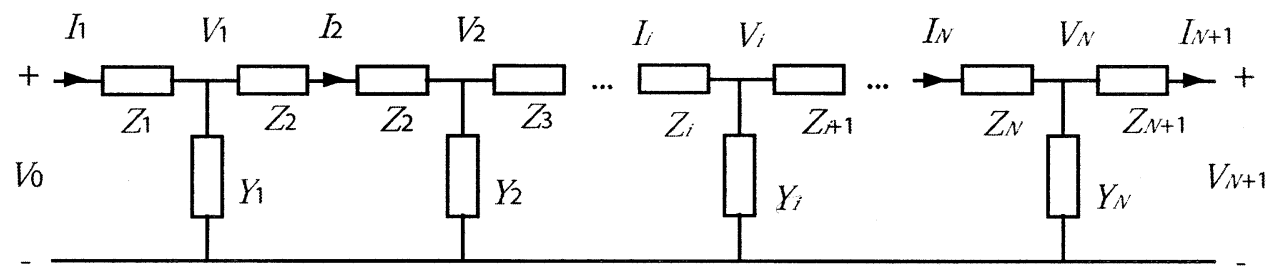

Fig. 2. FD-based lumped-element equivalent circuit.

and $a_{i j}$ 's are the DQ coefficients determined by the methods discussed previously. Taking a weighting summation of all the numerical derivatives in (8), and employing Theorem 1, it follows:

$$
a u_{1}^{\prime}+b u_{2}^{\prime}+c u_{3}^{\prime}+b u_{4}^{\prime}+a u_{5}^{\prime}=p u_{1}+q u_{2}-q u_{4}-p u_{5}
$$

where $a, b$ and $c$ are coefficients to be determined, and

$$
\begin{aligned}
& p=a a_{11}+b a_{21}+c a_{31}+b a_{41}+a a_{51} \\
& q=a a_{12}+b a_{22}+c a_{32}+b a_{42}+a a_{52} .
\end{aligned}
$$

Let $b=0, p=0$ and $q=-1$, then the coefficients are calculated by

$$
\begin{aligned}
a & =\frac{a_{31}}{a_{11} a_{32}+a_{32} a_{51}-a_{12} a_{31}-a_{31} a_{52}} \\
c & =\frac{a_{11}+a_{51}}{a_{12} a_{31}+a_{31} a_{52}-a_{11} a_{32}-a_{32} a_{51}} .
\end{aligned}
$$

Therefore, the DQM-based approximation frame for distributed voltages along the transmission lines isobtained as follows:

$$
u_{4}-u_{2}=a u_{1}^{\prime}+c u_{3}^{\prime}+a u_{5}^{\prime} .
$$

At the end points, the approximation framework (10) are not applicable. In this case, assume the left-end approximation framework have the following form:

$$
\begin{aligned}
& u_{2}-u_{1}=b_{1} u_{1}^{\prime}+a u_{3}^{\prime} \\
& u_{3}-u_{1}=b_{2} u_{2}^{\prime}+a u_{4}^{\prime} .
\end{aligned}
$$

We use the test functions (3) and (5) to determine the coefficients $b_{1}$ and $b_{2}$, corresponding to PDQ and HDQ, respectively. Let (11) be exact to as many functions in (3) or (5) as possible. Specificly, in this case, enforce (11) being exact when the first two test functions in (3) or (5) are taken, the coefficients can therefore be determine as

$$
b_{1}=\frac{1}{4}-a, b_{2}=\frac{1}{2}-a
$$

for PDQ, and

$$
b_{1}=\frac{\sqrt{2}}{2 \pi}, b_{2}=a+\frac{\sqrt{2}}{\pi}
$$

for HDQ.

Apparently, the right-end approximation framework can be analogously obtained

$$
\begin{aligned}
& u_{5}-u_{4}=a u_{3}^{\prime}+b_{1} u_{5}^{\prime} \\
& u_{5}-u_{3}=a u_{2}^{\prime}+b_{2} u_{4}^{\prime} .
\end{aligned}
$$

Equations (10), (11), and (14) constitute the complete approximation framework.

\section{Discrete Modeling TeChnique}

In this section, the approximation framework in (10), (11), and (14) are employed to develop the transmission line model. We begin with the simplest case of single line in this section, and then generalize the procedure to multiconductor transmission lines (MTL) in Section IV. In order to retain clarity of physical meaning, we follow the simplest conventional lumped-element equivalent circuit model based on the FD method, and then improve it by using the approximation frames of DQM-based modeling technique obtained in Section II.

\section{A. Conventional FD-Based Discrete Model}

Assume that a single transmission line stretches from 0 to $d$ along the $x$ axis of a Cartesian coordinate system, where $d$ is the length of the line. Let the distributed per-unit-length (PUL) parameters of the line be denoted by $R, L, G$, and $C$ representing resistance, inductance, conductance, and capacitance, respectively. The Telegrapher's equations can be written as

$$
\begin{aligned}
& \frac{d}{d x} V(x, s)=-(s L+R) I(x, s) \\
& \frac{d}{d x} I(x, s)=-(s C+G) V(x, s) .
\end{aligned}
$$

By making an FD approximation to the derivatives in (15), the discretization with respect to the space coordinate $x$ is equivalent to a lumped-element network [12]. Since there are different FD frameworks such as forward/backward difference and central difference methods, the equivalent lumped-element circuits may have different forms featuring T-cells, $\pi$-cells or half-cells. If the cell lengths are small enough, then the various models yield similar results [19]. By approximating the transmission line by a network of T-cells, a lumped-element circuit of a single line is shown by Fig. 2 .

In this equivalent circuit the voltage and current nodes are separated by a distance, which is in contrast to the alternatives where the samples of both voltages and currents are taken at the same points along the transmission lines. From Fig. 2, such a discrete frame has clear physical meaning featuring $R L C$ elements. Assuming the number of T-cells is $N$, the transmission line is segmented into $2 N$ sections, which form an open-loop circuit having $(2 N+1)$ nodes and $(2 N+3)$ state variables. Among the state variables are $(N+2)$ nodal voltages and $(N+1)$ inductance currents, which have the following relationship:

$$
\begin{gathered}
V_{i+1}-V_{i}=2 Z_{i} I_{i} \quad i=2, \ldots, N \\
I_{i+1}-I_{i}=Y_{i} V_{i} \quad i=1, \ldots, N
\end{gathered}
$$

for center grid points and

$$
\begin{aligned}
V_{2}-V_{1} & =Z_{1} I_{1} \\
V_{N+1}-V_{N} & =Z_{N+1} I_{N+1}
\end{aligned}
$$




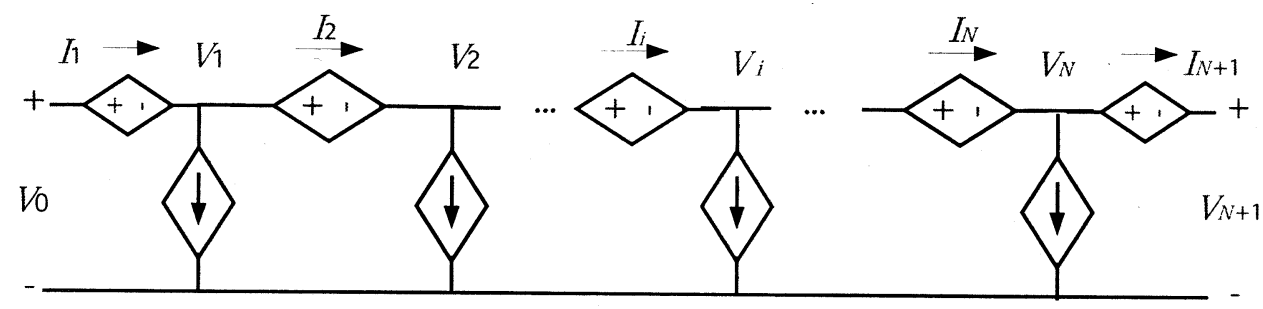

Fig. 3. Fifth-order DQM-based equivalent circuit.

for boundary points, where

$$
Z_{i}=\frac{(R+s L) d}{2 N}, Y_{i}=\frac{(G+s C) d}{N} .
$$

However, the choice of cell length depends on the minimum wavelength of interest as well as the electrical length of the transmission lines, which require that dozens of grid points per minimum wavelength are needed to achieve necessary computational accuracy. Consequently, the number of state variables increases to a great extent and the computational efficiency decreases remarkably. The applicability of this method is challenged especially when handling MTLs.

\section{B. Fifth-Order DQM-Based Discrete Model}

The fifth-order DQM-based discrete model can be obtained by analogy to the FD approximation framework in (16) and (17). Like in Fig. 2, the transmission line is uniformly segmented into $2 N$ sections, then the discrete circuit has $(2 N+1)$ nodes. At each node, the Telegrapher's equation is discretized as

$$
V_{i}^{\prime}=Z_{i} I_{i}, \quad I_{i}^{\prime}=Y_{i} V_{i} \quad i=1, \ldots,(2 N+1) .
$$

In order to apply DQM approximation framework, the length of transmission line should be normalized to match with the condition of (8). Specifically, the length of each small section is normalized to be $1 / 4$ unit so that five consecutive grid points constitute one-unit length over which the fifth-order DQM can be performed. Therefore, it follows in the discretized (19):

$$
\begin{aligned}
Z_{i} & =R_{i}+s L_{i}=\frac{4 d}{2 N}(R+s L) \\
Y_{i} & =G_{i}+s C_{i}=\frac{4 d}{2 N}(G+s C) i=1, \ldots,(2 N+1)
\end{aligned}
$$

Applying (10), (11), and (14) to the corresponding grid points, we obtain the DQM-based discrete modeling frameworks for voltage and current as represented by

$$
\begin{aligned}
V_{i+1}-V_{i}= & a Z_{i-1} I_{i}+c Z_{i} I_{i+1}+a Z_{i} I_{i+2}, \\
& i=1, \ldots,(N-1) \\
I_{i+1}-I_{i}= & a Y_{i-1} V_{i-1}+c Y_{i} V_{i}+a Y_{i+1} V_{i+1} \\
& i=2, \ldots,(N-1)
\end{aligned}
$$

for center grid points and

$$
\begin{aligned}
V_{1}-V_{0} & =b_{1} Z_{1} I_{1}+a Z_{2} I_{2} \\
V_{N+1}-V_{N} & =a Z_{N} I_{N}+b_{1} Z_{N+1} I_{N+1} \\
I_{2}-I_{1} & =b_{2} Y_{1} V_{1}+a Y_{2} V_{2} \\
I_{N+1}-I_{N} & =a Y_{N-1} V_{N-1}+b_{2} Y_{N} V_{N}
\end{aligned}
$$

for boundary points.
If defining the following current controlled voltage sources (CCVS)

$$
\begin{aligned}
V_{i}^{e} & =a Z_{i-1} I_{i}+c Z_{i} I_{i+1}+a Z_{i+1} I_{i+2}, \quad i=1, \ldots,(N-1) \\
V_{1}^{e} & =b_{1} Z_{1} I_{1}+a Z_{2} I_{2} \\
V_{N+1}^{e} & =a Z_{N} I_{N}+b_{1} Z_{N+1} I_{N+1}
\end{aligned}
$$

and the voltage controlled current sources (VCCS)

$$
\begin{aligned}
I_{i}^{e} & =a Y_{i-1} V_{i-1}+c Y_{i} V_{i}+a Y_{i+1} V_{i+1}, i=2, \ldots,(N-1) \\
I_{1}^{e} & =b_{2} Y_{1} V_{1}+a Y_{2} V_{2} \\
I_{N}^{e} & =a Y_{N-1} V_{N-1}+b_{2} Y_{N} V_{N}
\end{aligned}
$$

then the fifth-order DQM-based discrete model can be shown by Fig. 3 .

Fig. 3 shows that the DQM-based model has explicit physical meaning. The DQM-based discrete model is a chain comprised of CCVSs and VCCSs, compared to the FD-based discrete model that is a cascade of $R L C$ elements.

Assuming that $V_{0}$ and $V_{N+1}$ are, respectively, the applied voltages at two ports, the state equation of the open-loop discrete model shown in Fig. 3 can be formulated by using modified nodal analysis (MNA) [20]

$$
\left(\left[\begin{array}{cc}
\mathbf{P}_{1} & \mathbf{P}_{3} \\
-\mathbf{P}_{3}^{T} & \mathbf{P}_{2}
\end{array}\right]+s\left[\begin{array}{cc}
\mathbf{Q}_{1} & \mathbf{0} \\
\mathbf{0} & \mathbf{Q}_{2}
\end{array}\right]\right)\left[\begin{array}{l}
\mathbf{V} \\
\mathbf{I}
\end{array}\right]=\mathbf{b}\left[\begin{array}{c}
V_{0} \\
V_{N+1}
\end{array}\right]
$$

where $\mathbf{V}=\left[\begin{array}{llll}V_{1} & V_{2} & \ldots & V_{N}\end{array}\right]^{T} \in R^{N}$ is the vector of nodal voltages; $\mathbf{I}=\left[\begin{array}{lllll}I_{1} & I_{2} & \ldots & I_{N+1}\end{array}\right]^{T} \in R^{N+1}$ is the vector of branch currents; matrices $\mathbf{P}_{1} \in R^{N \times N}, \mathbf{P}_{2} \in R^{(N+1) \times(N+1)}$, $\mathbf{P}_{3} \in R^{N \times(N+1)}, \mathbf{Q}_{1} \in R^{N \times N}$ and $\mathbf{Q}_{2} \in R^{(N+1) \times(N+1)}$ are, respectively

$$
\begin{aligned}
\mathbf{P}_{1} & =\left[\begin{array}{ccccc}
b_{1} G_{i} & a G_{i} & & & \\
a G_{i} & c G_{i} & a G_{i} & & \\
& \ddots & \ddots & \ddots & \\
& & a G_{i} & c G_{i} & a G_{i} \\
& & & a G_{i} & b_{1} G_{i}
\end{array}\right] \\
\mathbf{P}_{2} & {\left[\begin{array}{ccccc}
b_{2} R_{i} & a R_{i} & & & \\
a R_{i} & c R_{i} & a R_{i} & & \\
& \ddots & \ddots & \ddots & \\
& & a R_{i} & c R_{i} & a R_{i} \\
& & & a R_{i} & b_{2} R_{i}
\end{array}\right] } \\
\mathbf{P}_{3} & {\left[\begin{array}{ccccc}
-1 & 1 & & \\
& -1 & 1 & & \\
& & \ddots & \ddots & \\
& & & -1 & 1
\end{array}\right] }
\end{aligned}
$$




$$
\begin{aligned}
& \mathbf{Q}_{1}=\left[\begin{array}{ccccc}
b_{1} C_{i} & a C_{i} & & & \\
a C_{i} & c C_{i} & a C_{i} & & \\
& \ddots & \ddots & \ddots & \\
& & a C_{i} & c C_{i} & a C_{i} \\
& & & a C_{i} & b_{1} C_{i}
\end{array}\right] \\
& \mathbf{Q}_{2}=\left[\begin{array}{ccccc}
b_{2} L_{i} & a L_{i} & & & \\
a L_{i} & c L_{i} & a L_{i} & & \\
& \ddots & \ddots & \ddots & \\
& & a L_{i} & c L_{i} & a L_{i} \\
& & & a L_{i} & b_{2} L_{i}
\end{array}\right] \\
& \mathbf{b}=\left[\begin{array}{cccccc}
0 & \ldots & 0 & 1 & \ldots & 0 \\
0 & \ldots & 0 & 0 & \ldots & -1
\end{array}\right] \text {. }
\end{aligned}
$$

\section{Passivity of the DQM-Based Model}

As stated in classical circuit theory, interconnections of stable systems may not necessarily be stable; interconnections of passive circuits are passive and therefore stable. When multiport models are connected together, the resulting overall circuit can guarantee to be stable only if each of the multiport models is passive [11]. In this view, it is extremely important to investigate the passivity of the discrete model that results from the discretization of telegrapher's equations. In order to do this, the following definitions and results are referred to [21].

Lemma 1: Necessary and sufficient conditions for a transfer function $n \times n$ matrix $\mathbf{Y}(s)$ to be passive is that $\mathbf{Y}(s)$ is positive-real: (1) each element of $\mathbf{Y}(s)$ is analytic in $\Re(s)>0$, (2) $\mathbf{Y}\left(s^{*}\right)=\mathbf{Y}^{*}(s)$, and $(3)\left(\mathbf{Y}^{*}\right)^{T}(s)+\mathbf{Y}(s)$ is nonnegative definite for all $\Re(s) \geq 0$.

Lemma 2: An $n$-port network is passive if and only if its admittance matrix $\mathbf{Y}(s)$ is positive-real.

Lemma 3: If $\mathbf{A}(s)$ is positive-real, then $\mathbf{A}^{-1}(s)$ is positivereal, if existed.

Lemma 4: If $\mathbf{A}(s)$ is positive-real and $\mathbf{B}$ is real, then $\mathbf{B}^{T} \mathbf{A}(s) \mathbf{B}$ is positive-real.

We now return to the MNA formed state equations (25). Noting that the original port variables are $V_{0}, I_{1}, V_{N+1}$, and $I_{N+1}$, the admittance matrix is obtained as

$$
\mathbf{Y}(s)=\mathbf{b}^{T}\left(\left[\begin{array}{cc}
\mathbf{P}_{1} & \mathbf{P}_{3} \\
-\mathbf{P}_{3}^{T} & \mathbf{P}_{2}
\end{array}\right]+s\left[\begin{array}{cc}
\mathbf{Q}_{1} & \mathbf{0} \\
\mathbf{0} & \mathbf{Q}_{2}
\end{array}\right]\right)^{-1} \mathbf{b}
$$

and the passivity of (32) is determined by the following theorem.

Theorem 2: The matrix $\mathbf{Y}(s)$ in (32) is positive-real.

Proof: Using Lemmas 1-4, the matrix $\mathbf{Y}(s)$ being positive-real ascribes to that the following matrix is positive-real

$$
\mathbf{W}=\left[\begin{array}{cc}
\mathbf{P}_{1} & \mathbf{P}_{3} \\
-\mathbf{P}_{3}^{T} & \mathbf{P}_{2}
\end{array}\right]+s\left[\begin{array}{cc}
\mathbf{Q}_{1} & \mathbf{0} \\
\mathbf{0} & \mathbf{Q}_{2}
\end{array}\right]
$$

Referring back to Lemma 1, the first two conditions are automatically satisfied for matrix $\mathbf{W}$. In proving that matrix $\mathbf{W}$ satisfies condition (3), noting that matrices $\mathbf{P}_{1}, \mathbf{P}_{2}, \mathbf{Q}_{1}$, and $\mathbf{Q}_{2}$ are all symmetric, it follows

$$
\mathbf{W}+\left(\mathbf{W}^{*}\right)^{T}=2\left[\begin{array}{cc}
\mathbf{P}_{1} & \mathbf{0} \\
\mathbf{0} & \mathbf{P}_{2}
\end{array}\right]+2 \Re(s)\left[\begin{array}{cc}
\mathbf{Q}_{1} & \mathbf{0} \\
\mathbf{0} & \mathbf{Q}_{2}
\end{array}\right] .
$$

As matrices $\mathbf{P}_{1}, \mathbf{P}_{2}, \mathbf{Q}_{1}$, and $\mathbf{Q}_{2}$ are all nonnegative, $\mathbf{W}+$ $\left(\mathbf{W}^{*}\right)^{T}$ is therefore nonnegative. Thus, the matrix $\mathbf{Y}(s)$ is positive-real and the DQM-based model for single transmission line preserves passivity.
Some remarks on DQM modeling technique and passivity are as follows. (a) As a general rule, cast into MNA equation like (25), a circuit is passive if the matrices $\mathbf{P}_{1}, \mathbf{P}_{2}, \mathbf{Q}_{1}$, and $\mathbf{Q}_{2}$ are all nonnegative and symmetric. An immediate result of this rule is that the discrete modeling shown in Fig. 2 is also passive. (b) The symmetry of the matrices $\mathbf{P}_{1}, \mathbf{P}_{2}, \mathbf{Q}_{1}$, and $\mathbf{Q}_{2}$ is guaranteed by uniformity of transmission line, and boundary approximation frames (11) and (14). (c) In (25), matrices $\mathbf{P}_{3}$ and $-\mathbf{P}_{3}^{T}$ are naturally formulated by modified nodal analysis, as stated in [22]. (d) With comparable accuracy, the DQM-based modeling has higher efficiency than FD-based modeling, which is shown in Section V. The reason is that the proposed modeling approach employs the global approximation framework, which leads to the fact that the matrices $\mathbf{P}_{1}, \mathbf{P}_{2}, \mathbf{Q}_{1}$, and $\mathbf{Q}_{2}$ for DQMbased modeling are denser than those for FD-based modeling: the former are tridiagonal matrices, and the latter are diagonal matrices.

\section{Discrete Modeling of Multiconductor TRANSMISSION LINES}

\section{A. Discrete Model}

The discrete model of multiconductor transmission lines (MTL) can be straightforward obtained by extending that of single transmission line. Similarly, the MTL is segmented into $2 N$ sections. At each grid point, the Telegrapher's equations of $M$-coupled transmission lines are represented by

$$
\mathbf{V}_{i}^{\prime}=\mathbf{Z}_{i} \mathbf{I}_{i}, \quad \mathbf{I}_{i}^{\prime}=\mathbf{Y}_{i} \mathbf{V}_{i}, \quad i=1, \ldots,(2 N+1)
$$

where

$$
\begin{aligned}
\mathbf{V}_{i} & =\left[V_{i}^{1} V_{i}^{2} \ldots V_{i}^{M}\right]^{T} \\
\mathbf{I}_{i} & =\left[I_{i}^{1} I_{i}^{2} \ldots I_{i}^{M}\right]^{T}
\end{aligned}
$$

are voltage and current vectors corresponding to the $i$ th gird point, respectively, and

$$
\begin{aligned}
\mathbf{Z}_{i} & =\mathbf{R}_{i}+s \mathbf{L}_{i}=\frac{4 d}{2 N}(\mathbf{R}+s \mathbf{L}) \\
\mathbf{Y}_{i} & =\mathbf{G}_{i}+s \mathbf{C}_{i}=\frac{4 d}{2 N}(\mathbf{G}+s \mathbf{C}), i=1, \ldots,(2 N+1) .
\end{aligned}
$$

The approximation frames of MTL are as follows:

$$
\begin{aligned}
\mathbf{V}_{i+1}-\mathbf{V}_{i}= & a \mathbf{Z}_{i-1} \mathbf{I}_{i}+c \mathbf{Z}_{i} \mathbf{I}_{i+1}+a \mathbf{Z}_{i+2} \mathbf{I}_{i+1} \\
& i=1, \ldots,(N-1) \\
\mathbf{I}_{i+1}-\mathbf{I}_{i}= & a \mathbf{Y}_{i-1} \mathbf{V}_{i-1}+c \mathbf{Y}_{i} \mathbf{V}_{i}+a \mathbf{Y}_{i+1} \mathbf{V}_{i+1} \\
& i=2, \ldots,(N-1)
\end{aligned}
$$

for center grid points and

$$
\begin{aligned}
\mathbf{V}_{1}-\mathbf{V}_{0} & =b_{1} \mathbf{Z}_{1} \mathbf{I}_{1}+a \mathbf{Z}_{2} \mathbf{I}_{2} \\
\mathbf{V}_{N+1}-\mathbf{V}_{N} & =a \mathbf{Z}_{N} \mathbf{I}_{N}+b_{1} \mathbf{Z}_{N+1} \mathbf{I}_{N+1} \\
\mathbf{I}_{2}-\mathbf{I}_{1} & =b_{2} \mathbf{Y}_{1} \mathbf{V}_{1}+a \mathbf{Y}_{2} \mathbf{V}_{2} \\
\mathbf{I}_{N+1}-\mathbf{I}_{N} & =a \mathbf{Y}_{N-1} \mathbf{V}_{N-1}+b_{2} \mathbf{Y}_{N} \mathbf{V}_{N}
\end{aligned}
$$

for boundary points. Similarly define the VCCSs and CCVSs as in Fig. 3, the equivalent circuit for MTL can be shown as in Fig. 4. 


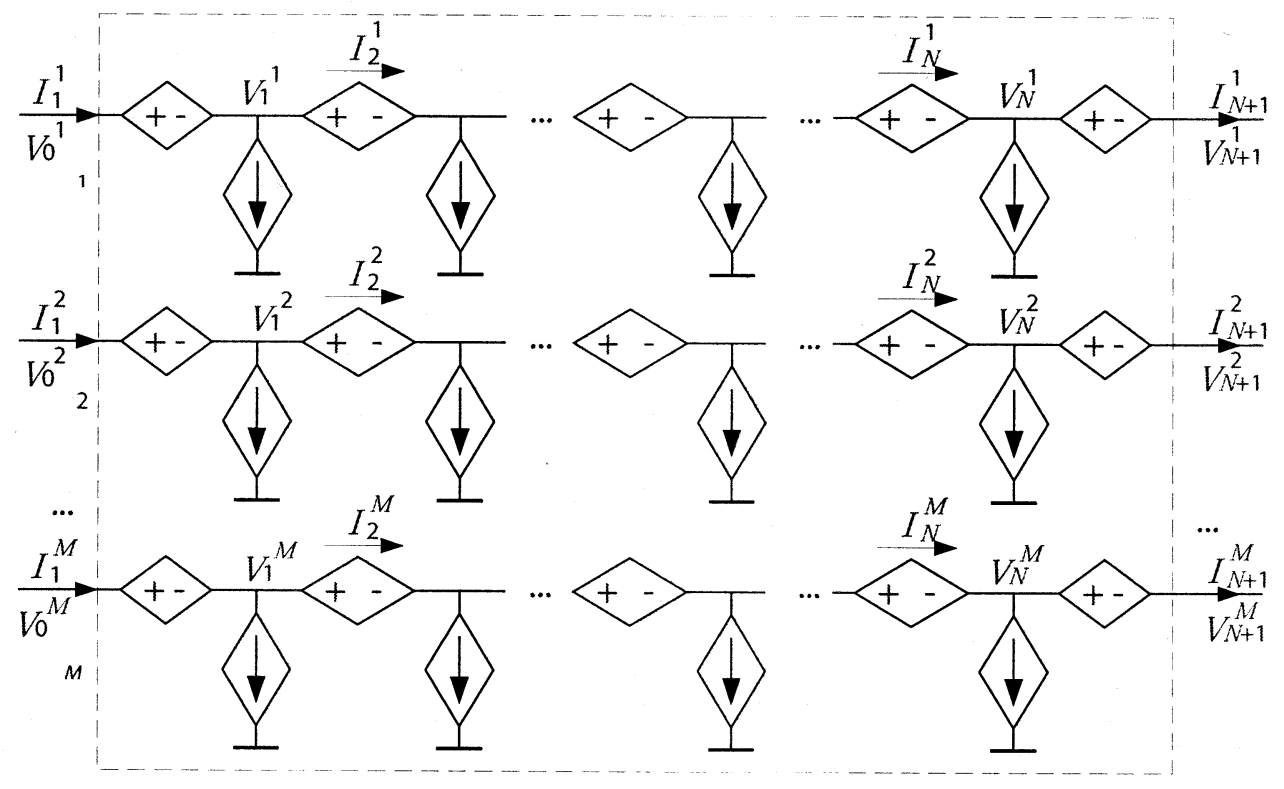

Fig. 4. Equivalent circuit of MTL.

Likewise, the open-loop MNA-formed state equation of the discrete model of an MTL follows:

$$
\left(\left[\begin{array}{cc}
\widehat{\mathbf{P}}_{1} & \widehat{\mathbf{P}}_{3} \\
-\widehat{\mathbf{P}}_{3}^{T} & \widehat{\mathbf{P}}_{2}
\end{array}\right]+s\left[\begin{array}{cc}
\widehat{\mathbf{Q}}_{1} & \mathbf{0} \\
\mathbf{0} & \widehat{\mathbf{Q}}_{2}
\end{array}\right]\right)\left[\begin{array}{c}
\widehat{\mathbf{V}} \\
\widehat{\mathbf{I}}
\end{array}\right]=\widehat{\mathbf{b}}\left[\begin{array}{c}
\widehat{\mathbf{V}}_{0} \\
\widehat{\mathbf{V}}_{N+1}
\end{array}\right]_{(38)}
$$

where $\widehat{\mathbf{V}}=\left[\begin{array}{llll}\mathbf{V}_{1}^{T} & \mathbf{V}_{2}^{T} \ldots \mathbf{V}_{N}^{T}\end{array}\right]^{T} \in R^{N M}$ is the vector of nodal voltages; $\hat{\mathbf{I}}=\left[\begin{array}{llll}\mathbf{I}_{1}^{T} & \mathbf{I}_{2}^{T} & \ldots & \mathbf{I}_{N+1}^{T}\end{array}\right]^{T} \in R^{(N+1) M}$ is the vector of branch currents; matrices $\widehat{\mathbf{P}}_{1} \in R^{N M \times N M}, \widehat{\mathbf{P}}_{2} \in$ $R^{(N+1) M \times(N+1) M}, \widehat{\mathbf{P}}_{3} \in R^{N M \times(N+1) M}, \widehat{\mathbf{Q}}_{1} \in R^{N M \times N M}$ and $\widehat{\mathbf{Q}}_{2} \in R^{(N+1) M \times(N+1) M}$ are, respectively

$$
\begin{aligned}
\widehat{\mathbf{P}}_{1} & =\left[\begin{array}{ccccc}
b_{1} \mathbf{G}_{i} & a \mathbf{G}_{i} & & & \\
a \mathbf{G}_{i} & c \mathbf{G}_{i} & a \mathbf{G}_{i} & & \\
& \ddots & \ddots & \ddots & \\
& & a \mathbf{G}_{i} & c \mathbf{G}_{i} & a \mathbf{G}_{i} \\
& & & a \mathbf{G}_{i} & b_{1} \mathbf{G}_{i}
\end{array}\right] \\
\widehat{\mathbf{P}}_{2} & =\left[\begin{array}{ccccc}
b_{2} \mathbf{R}_{i} & a \mathbf{R}_{i} & & & \\
a \mathbf{R}_{i} & c \mathbf{R}_{i} & a \mathbf{R}_{i} & & \\
& \ddots & \ddots & \ddots & \\
& & a \mathbf{R}_{i} & c \mathbf{R}_{i} & a \mathbf{R}_{i} \\
& & & a \mathbf{R}_{i} & b_{2} \mathbf{R}_{i}
\end{array}\right] \\
\widehat{\mathbf{P}}_{3} & =\left[\begin{array}{ccccc}
-\mathbf{U} & \mathbf{U} & & & \\
& -\mathbf{U} & \mathbf{U} & & \\
& & \ddots & \ddots & \\
& & & -\mathbf{U} & \mathbf{U}
\end{array}\right] \\
\widehat{\mathbf{Q}}_{1} & =\left[\begin{array}{lllll}
b_{1} \mathbf{C}_{i} & a \mathbf{C}_{i} & & & \\
a \mathbf{C}_{i} & c \mathbf{C}_{i} & a \mathbf{C}_{i} & & \\
& \ddots & \ddots & \ddots & \\
& & a \mathbf{C}_{i} & c \mathbf{C}_{i} & a \mathbf{C}_{i} \\
& & & a \mathbf{C}_{i} & b_{1} \mathbf{C}_{i}
\end{array}\right]
\end{aligned}
$$

$$
\begin{aligned}
\widehat{\mathbf{Q}}_{2} & =\left[\begin{array}{cccccc}
b_{2} \mathbf{L}_{i} & a \mathbf{L}_{i} & & & \\
a \mathbf{L}_{i} & c \mathbf{L}_{i} & a \mathbf{L}_{i} & & \\
& & \ddots & \ddots & \ddots & \\
& & & a \mathbf{L}_{i} & c \mathbf{L}_{i} & a \mathbf{L}_{i} \\
& & & & a \mathbf{L}_{i} & b_{2} \mathbf{L}_{i}
\end{array}\right] \\
\widehat{\mathbf{b}} & =\left[\begin{array}{cccccc}
\mathbf{0} & \ldots & \mathbf{0} & \mathbf{U} & \ldots & \mathbf{0} \\
\mathbf{0} & \ldots & \mathbf{0} & \mathbf{0} & \ldots & -\mathbf{U}
\end{array}\right]
\end{aligned}
$$

where $\mathbf{U}$ is the $M \times M$ unit matrix.

\section{B. Passivity}

Analogously, noting that the original port variables are $\mathbf{V}_{0}$, $\mathbf{I}_{1}, \mathbf{V}_{N+1}$ and $\mathbf{I}_{N+1}$, the admittance matrix for MTL is represented by

$$
\widehat{\mathbf{Y}}(s)=\widehat{\mathbf{b}}^{T}\left(\left[\begin{array}{cc}
\widehat{\mathbf{P}}_{1} & \widehat{\mathbf{P}}_{3} \\
-\widehat{\mathbf{P}}_{3}^{T} & \widehat{\mathbf{P}}_{2}
\end{array}\right]+s\left[\begin{array}{cc}
\widehat{\mathbf{Q}}_{1} & \mathbf{0} \\
\mathbf{0} & \widehat{\mathbf{Q}}_{2}
\end{array}\right]\right)^{-1} \widehat{\mathbf{b}} .
$$

The passivity of MTL discrete model is guaranteed by the positive-real property of (45), which is shown by the theorem below.

Theorem 3: The matrix $\widehat{\mathbf{Y}}(s)$ in (45) is positive-real.

Proof: Using Lemmas 1-4 and referring to the proof of the positive-real property of (32), this theorem can be proved similarly.

To complete this section, the quantitative statistic for the fifth-order DQM-based modeling of an $M$ coupled MTL is summed up as follows: the number of discrete sections is $2 N$, the number of nodes in the open loop circuit is $(2 N+1) M$, the number of voltage state variables is $(N+2) M$, the number of current state variables is $(N+1) M$, and the total number of state variables is thus $(2 N+3) M$. 
TABLE I

STANDARD ERRors ( $N$ : THE NUMBER OF SECTIONS)

\begin{tabular}{l||c|c|c|c|c|c|c|c}
\hline$N$ & 6 & 8 & 10 & 12 & 14 & 16 & 18 & 20 \\
\hline FD & 0.6752 & 0.2179 & 0.0896 & 0.0432 & 0.0233 & 0.0136 & 0.0085 & 0.0055 \\
\hline PDQ5 & 0.0268 & 0.0041 & 0.0018 & $6.0952 \mathrm{e}-4$ & $2.0443 \mathrm{e}-4$ & $7.4012 \mathrm{e}-5$ & $2.9120 \mathrm{e}-5$ & $1.2376 \mathrm{e}-5$ \\
\hline HDQ5 & 0.0176 & $1.0503 \mathrm{e}-12$ & 0.0225 & 0.0860 & 0.1572 & 0.2163 & 0.2595 & 0.2888 \\
\hline
\end{tabular}

\section{ACCURACY OF DQM-BASED DISCRETE MODELING TECHNIQUE}

In this section, we investigate the efficiency and accuracy of the proposed modeling approach. Similar to FD-based discrete model, DQM-based model leads to filter-like multiport devices. This kind of models have the approximate transfer functions which are agreeable to the exact ones over the low frequency band, and have more error as the frequency increases.

In practical applications, the accurate modeling means to guarantee its accuracy over a frequency bandwidth from 0 to a high frequency of interest. If the maximum frequency and the features of interconnections are known, the minimum wavelength can be simply obtained. The efficiency of discrete modeling technique is represented by the freedom degree (the number of grid points) within a minimum wavelength to achieve an expected accuracy. It is known that the number of grid points for FD modeling technique is needed to be as high as dozens. In order to evaluate the efficiency of the presented DQM-based modeling method, we compare it to FD method. As the bases of the presented method is (8), we will first determine how many grid points DQ approximation (8) needs over a minimum wavelength to achieve a comparable accuracy.

Assuming that a signal in Fourier analysis has the form of sine function along the minimum wavelength and that the wavelength is normalized to be 1, e.g., $f(x)=\sin 2 \pi x, x \in[0,1]$, we use FD, fifth-order PDQ, and fifth-order HDQ to compute the numerical differentiations and compare their standard errors. The unit wavelength is equally segmented into smaller sections, over which the FD and DQ approximations are performed. The results are shown as in Table I.

From Table I, the error of FD method keeps decreasing as the number of segments increases. This also occurs to that of PDQ method, but at a faster rate. The error of HDQ, however, shows oscillation as the number of sections increases. In order to achieve most accuracy as well as efficiency, we consider fifth-order PDQ approximation adopting eight equal sections and fifth-order HDQ approximation adopting eight equal sections. From Table I, both approximation approaches can give comparable accuracy to FD method with 20 sections. Their numerical differentiations over a unit minimum wavelength are shown in Fig. 5, where central difference FD, fifth-order HDQ and fifth-order PDQ use 20, eight, and eight equal sections, respectively.

From the above comparison of per minimum wavelength resolution, a heuristic rule for the resolution of fifth-order DQMs is shown to segment eight equal sections per minimum wavelength in the spectrum. Therefore, for a transmission line with length $d$, the number of sample points for fifth-order DQM based modeling technique is

$$
N_{p}=\frac{8 d}{\lambda_{\min }}+1
$$

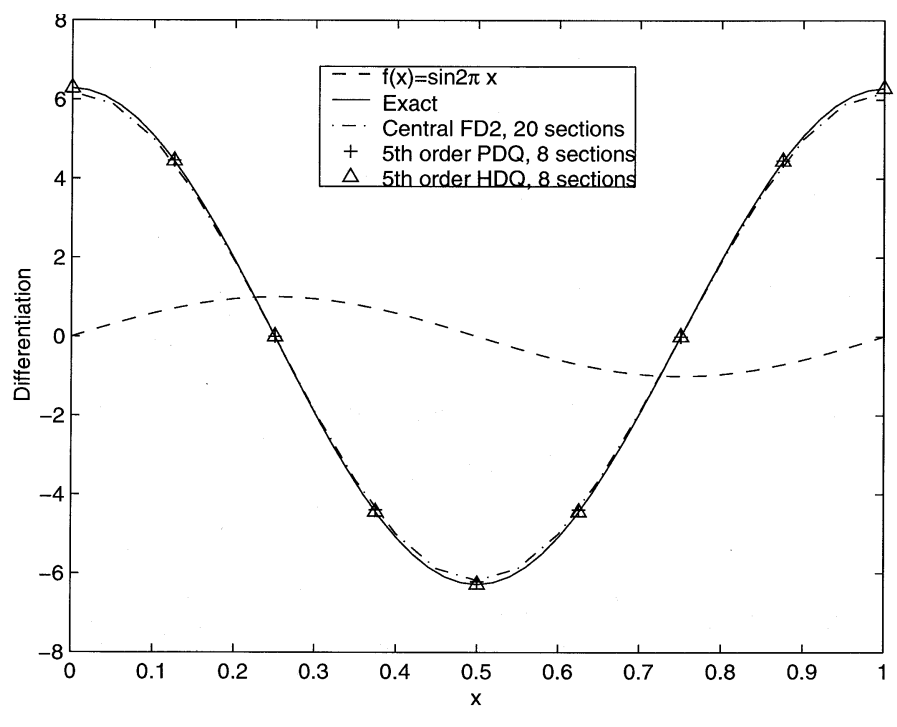

Fig. 5. Numerical differentiations over a minimum wavelength.

where $\lambda_{\min }$ is the minimum wavelength. Accordingly, the number of state variables of discrete model of a line with length $d$ is $\left(N_{p}+3\right)$ for DQ modeling.

For different standards, there are different methods to determine the maximum high frequency. Here we refer to a metric adopted by HSPICE [23], the maximum frequency of interest can be evaluated by

$$
f_{\max }=\frac{0.35}{t_{r}}
$$

where $t_{r}$ is the rise time of the input waveform. The maximum frequency determines the minimum wavelength within the spectral range of interest.

\section{Circuit Formulation AND ApPlications}

The frequency-domain models presented in previous sections can be directly incorporated in formulating reduction algorithm like PRIMA [11]. However, this paper focuses on the efficiency and accuracy of the discrete modeling technique, and will not be concerned on reduction models. Instead, the presented models are directly incorporated into MNA matrix. Following the approach in the literature [24], the time-domain counterparts of discrete transmission line model described by (25) and (38) can be directly incorporated into MNA equations as stamps. Next we present several examples. All the fifth-order PDQ and fifth-order HDQ used in these examples employ equally spaced grid points. Since the spatial distributions of the grid points have been fixed, all the coefficients in the approximation frames used in these examples are available constants which have been already obtained by the approaches of Section II. 


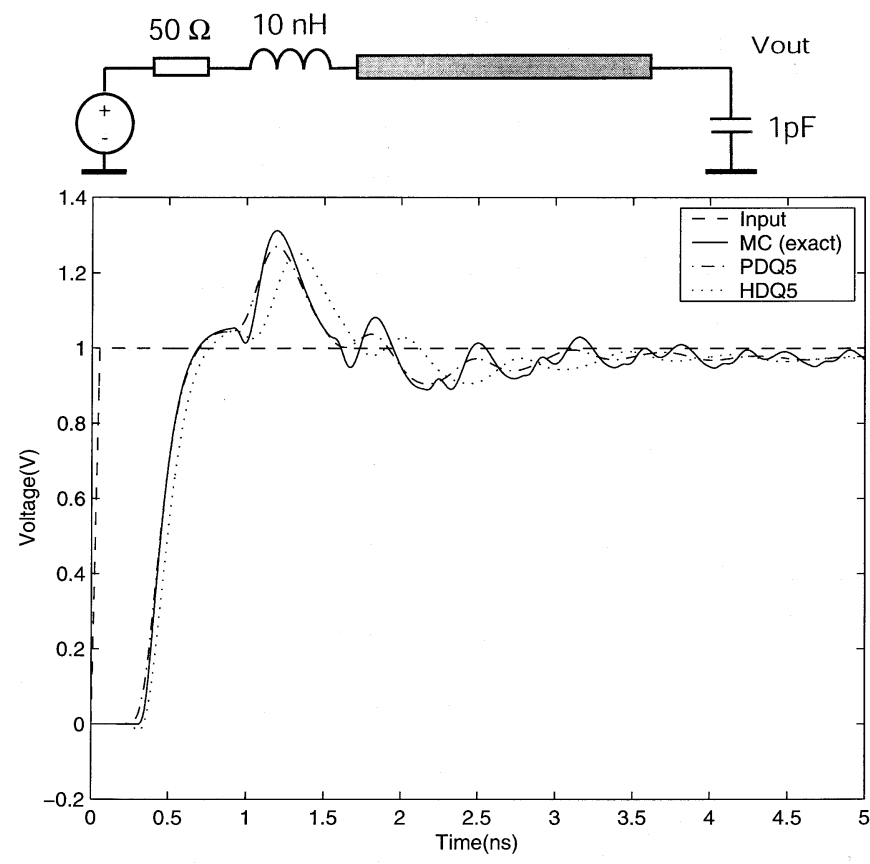

Fig. 6. Transient responses of single transmission line.

The first example, a single transmission line as shown in Fig. 6, shows the transient simulation accuracy of the presented modeling technique. The length of this transmission line is $5 \mathrm{~cm}$, and the PUL parameters are $l=360, c=100, r=36$ and $g=0.01$ (Hereinafter, the units of PUL parameters are $\mathrm{nH} / \mathrm{m}$, $\mathrm{pF} / \mathrm{m}, \Omega / \mathrm{m}$, and $\mathrm{S} / \mathrm{m}$ for inductance, capacitance, resistance, and conductance, respectively). Noting that the transmission line in this example is an undistorted line, the transient simulation response calculated by the Method of Characteristics is exact value if the round errors are neglected [25].

Assume that the applied input is a step voltage whose rise time is $50 \mathrm{ps}$. The propagation velocity along the line is $5 / 3 \times 10^{8} \mathrm{~m} / \mathrm{s}$, and by (47) the maximum frequency is $f_{\max }=7 \mathrm{GHz}$. Equation (46) shows that the number of sections using fifth-order DQ methods is approximately calculated as 8. Therefore, the number of state variables is 11. Fig. 6 shows that the transient result by PDQ is most agreeable with the exact value.

The second example consists of three coupled transmission lines as shown in Fig. 7. The length of each transmission line is $4 \mathrm{~cm}$, and its RLCG parameters are: $R_{11}=R_{22}=R_{33}=$ 344.8, $L_{11}=L_{22}=L_{33}=497.6, L_{12}=L_{23}=76.5, L_{13}=0$, $C_{11}=C_{33}=108.2, C_{22}=112.4, C_{12}=C_{23}=-19.7, C_{13}=0$, $G_{11}=G_{22}=G_{33}=0.1, G_{12}=G_{23}=-0.01$, and $G_{33}=0$.

Input excitation is a trapezoidal pulse with $100 \mathrm{ps}$ rise/fall time whose magnitude is $1 \mathrm{~V}$. The phase velocities along the transmission lines are obtained by eigenvalue analysis of $\mathbf{L C}$ as following, $v_{p 1}=1.4184 \times 10^{8} \mathrm{~m} / \mathrm{s}, v_{p 2}=1.3625 \times 10^{8} \mathrm{~m} / \mathrm{s}$ and $v_{p 3}=1.3601 \times 10^{8} \mathrm{~m} / \mathrm{s}$, respectively. According to the rule of (47), the highest frequency of interest is $3.5 \mathrm{GHz}$. The minimum wavelength is thus calculated as $3.89 \mathrm{~cm}$. Application of (46) shows that the number of sections using fifth-order DQ method is approximately calculated as 8 . Therefore, the number of state variables of transmission line for either PDQ or HDQ

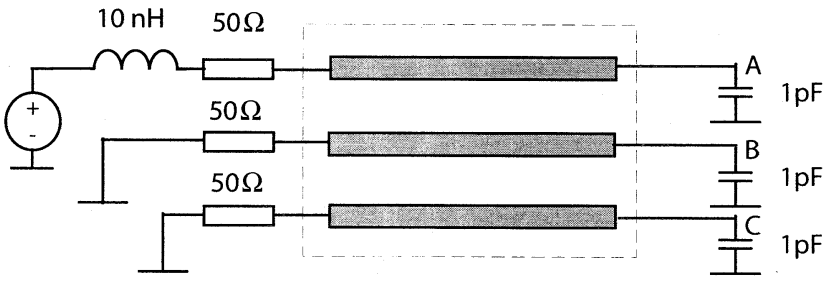

Fig. 7. Circuit of 3-coupled transmission line.

is $11 \times 3=33$. The main line waveform at point $A$ and the crosstalk waveform at point $B$ are shown in Fig. 8, altogether with the result of HSPICE. The accuracy of transmission line modeling is usually represented better by the accuracy of the transient results at victim line. The simulation results show both DQM-based modeling and HSPICE are agreeable.

In this example, HSPICE uses the FD method and segments the transmission line into 20 sections, while the DQ methods need only 8 sections. Furthermore, the 20 sections of FD method lead to $42 \times 3=126$ state variables, while the 8 sections of DQM modeling lead to only $11 \times 3=33$ state variables, as stated at the end of Section IV. The whole circuit has 29 nodes using the proposed modeling technique, in contrast to 89 nodes in using HSPICE. Cast in MNA matrices, the state equations of the proposed methods have the size of $33 \times 33$, while that of FD method has the size of $126 \times 126$. As the running time of solving $n \times n$ linear equations is generally proportional to $n^{3}$, the fifth-order DQMs have as dozens times efficiency as FD method to obtain accurate discrete modeling. This estimate is basically verified by the total running time on an Ultra-1 SUN workstation: taking the same time step, the running time by DQM modeling is $0.108 \mathrm{~s}$, while that of HSPICE is $0.7 \mathrm{~s}$.

The third example consists of coupled transmission lines as shown in Fig. 9. Among the lumped elements of this example, each of the resistors has the value of $50 \Omega$, each of the capacitor has the value of $1 \mathrm{pF}$, and the inductance is $10 \mathrm{nH}$. The length of both coupled transmission lines is $5 \mathrm{~cm}$. The distributed parameters of the two-coupled line (TL1) are represented in the following matrices:

$$
\begin{aligned}
& \mathbf{L}=\left[\begin{array}{cc}
494.6 & 63.3 \\
63.3 & 494.6
\end{array}\right], \quad \mathbf{C}=\left[\begin{array}{cc}
62.8 & -4.9 \\
-4.9 & 62.8
\end{array}\right] \\
& \mathbf{R}=\left[\begin{array}{cc}
100 & 0.0 \\
0.0 & 100
\end{array}\right], \mathbf{G}=\left[\begin{array}{cc}
0.1 & -0.01 \\
-0.01 & 0.1
\end{array}\right]
\end{aligned}
$$

The four-coupled line (TL2) has the following distributed parameters:

$$
\begin{aligned}
\mathbf{L} & =\left[\begin{array}{cccc}
494.6 & 63.3 & 7.8 & 0.0 \\
63.3 & 494.6 & 63.3 & 7.8 \\
7.8 & 63.3 & 494.6 & 63.3 \\
0.0 & 7.8 & 63.3 & 494.6
\end{array}\right] \\
\mathbf{C} & =\left[\begin{array}{cccc}
62.8 & -4.9 & -0.3 & 0.0 \\
-4.9 & 62.8 & -4.9 & -0.3 \\
-0.3 & -4.9 & 62.8 & -4.9 \\
0.0 & -0.3 & -4.9 & 62.8
\end{array}\right] \\
\mathbf{R} & =\left[\begin{array}{cccc}
100 & 0.0 & 0.0 & 0.0 \\
0.0 & 100 & 0.0 & 0.0 \\
0.0 & 0.0 & 100 & 0.0 \\
0.0 & 0.0 & 0.0 & 100
\end{array}\right]
\end{aligned}
$$




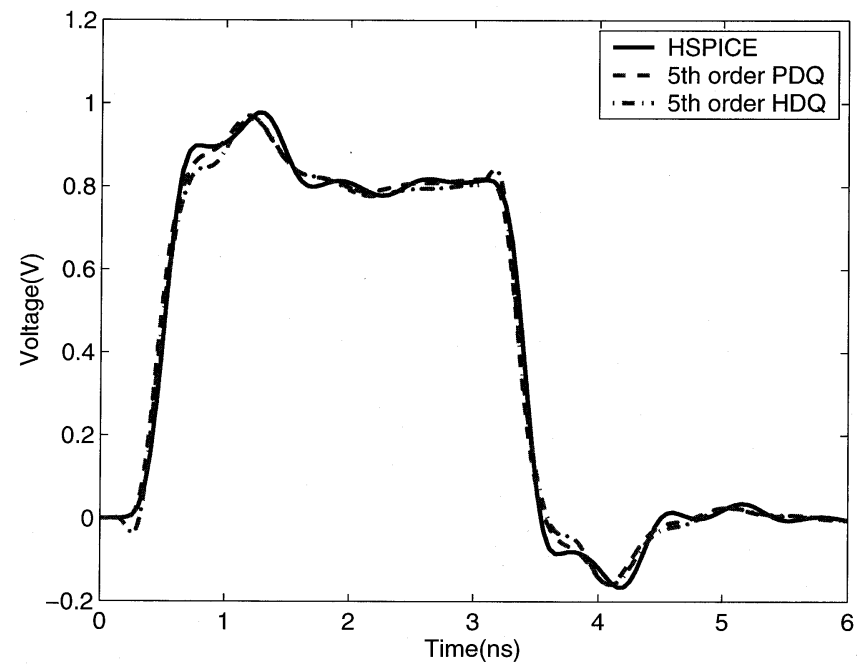

(a)

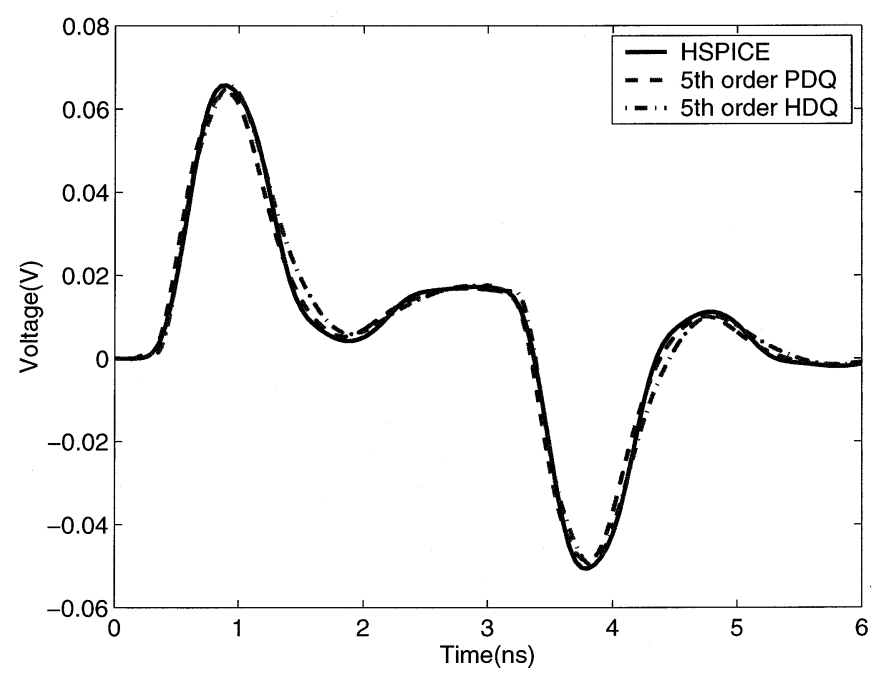

(b)

Fig. 8. Transient responses at (a) point A and (b) point B in Fig. 7.

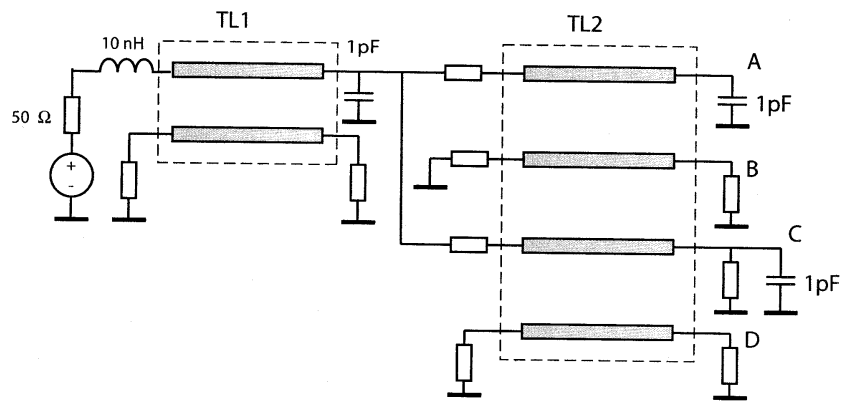

Fig. 9. MTL network.

$$
\mathbf{G}=\left[\begin{array}{cccc}
.1 & -.01 & -.001 & 0.0 \\
-.01 & .1 & -.01 & -.001 \\
-.001 & -.01 & .1 & -.01 \\
0.0 & -.001 & -.01 & .1
\end{array}\right]
$$

The phase velocities along the transmission lines are obtained by eigenvalue analysis of $L C$. For the TL1, the phase velocities of two modes are $v_{p 1}=1.8506 \times 10^{8} \mathrm{~m} / \mathrm{s}$ and $v_{p 2}=1.7595 \times$ $10^{8} \mathrm{~m} / \mathrm{s}$, respectively; for the TL2, they are $v_{p 1}=1.8859 \times$

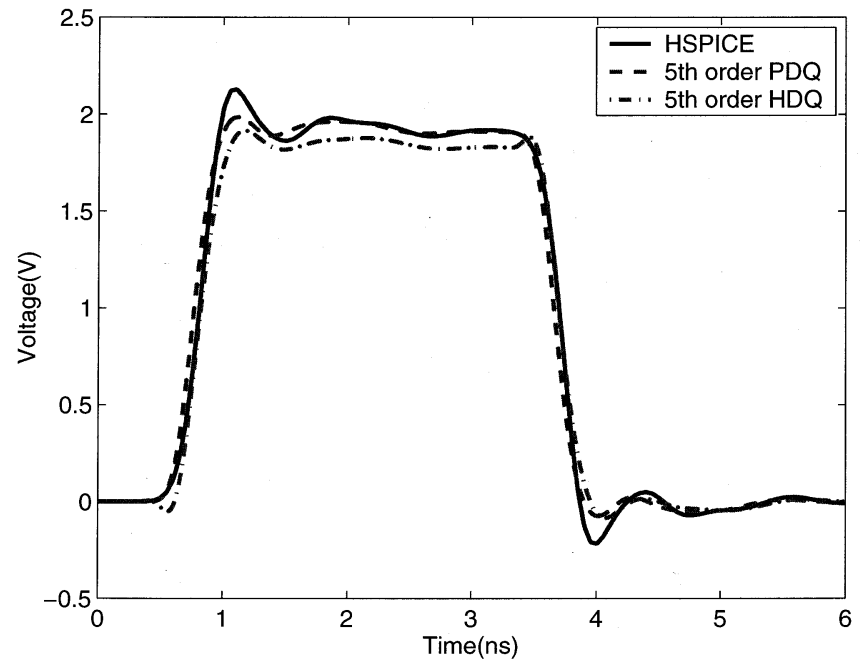

(a)

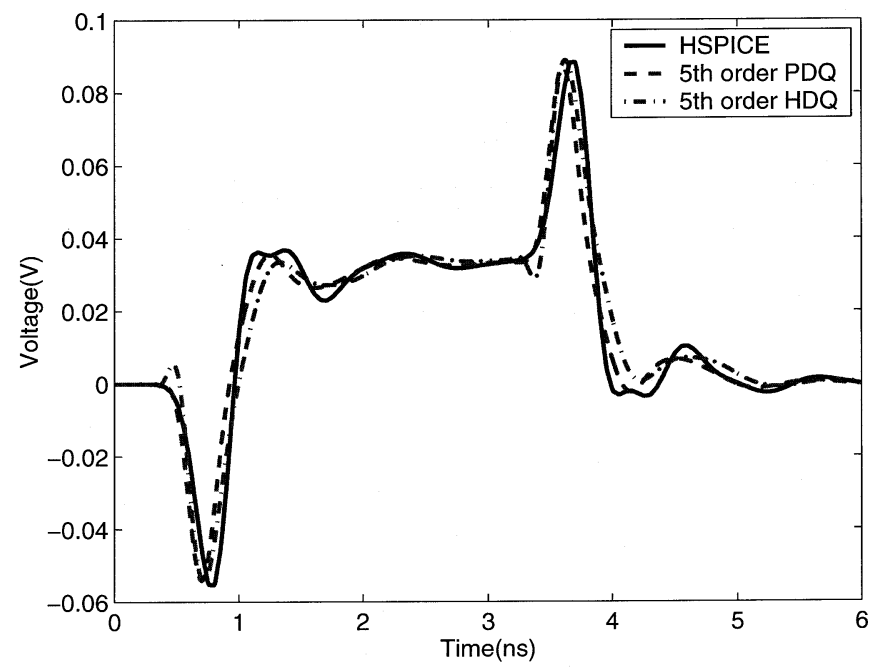

(b)

Fig. 10. Transient responses at (a) point A and (b) point B Fig. 9.

TABLE II

Comparison of Circuit Structures of Discrete Modeling Technique

\begin{tabular}{l||c|c|c}
\hline Quantitative descriptions & PDQ & HDQ & HSPICE \\
\hline \hline Number of discrete sections of TL1 & 8 & 8 & 20 \\
\hline Number of discrete sections of TL2 & 8 & 8 & 20 \\
\hline Number of total nodes & 56 & 56 & 175 \\
\hline Number of total state variables & 66 & 66 & 252 \\
\hline Total CPU time (s) & 0.54 & 0.54 & 1.55 \\
\hline
\end{tabular}

$10^{8} \mathrm{~m} / \mathrm{s}, v_{p 2}=1.7412 \times 10^{8} \mathrm{~m} / \mathrm{s}, v_{p 3}=1.8369 \times 10^{8} \mathrm{~m} / \mathrm{s}$, and $v_{p 4}=1.7780 \times 10^{8} \mathrm{~m} / \mathrm{s}$.

If the excitation is a pulse with rise/fall time $0.1 \mathrm{~ns}$ and $2.8 \mathrm{~ns}$ square width, the highest frequency of interest will be $f_{\max }=$ $3.5 \mathrm{GHz}$. Then the minimum wavelength for the TL1 is $\lambda_{\min }=$ $v_{p 2} / f_{\max }=5.027 \mathrm{~cm}$, and the minimum wavelength for the TL2 is $4.975 \mathrm{~cm}$. The transient responses of main transmission lines and the responses of victim lines are shown in Fig. 10.

The statistic data in solving this example by the proposed modeling technique and HSPICE are compared in Table II. Taking the same time step, the total running time on ULTRA-1 
SUN workstation is $0.54 \mathrm{~s}$ for the fifth-order DQM-based modeling technique and $1.55 \mathrm{~s}$ for HSPICE.

\section{CONCLUSION}

A numerical technique called the DQM is adapted for interconnect modeling. The approximation framework is derived based on the fifth-order DQM, and its advantageous computational accuracy and efficiency are demonstrated in contrast to those of FD methods. In the $s$-domain, the fifth-order DQM-based modeling methods represent transmission lines by matrix equations which is compatible with Krylov subspace techniques for circuit reduction. The proposed modeling approaches generate equivalent-circuit interconnect models consisting of VCCSs and CCVSs, which can be directly incorporated into circuit simulators like SPICE. The proposed modeling approaches have been shown to produce highly efficient equivalent-circuit models. For both single and multiconductor transmission lines, the fifth-order DQM-based modeling technique generates as 8/20 discrete sections, 9/21 circuit nodes, and 11/42 state variables as HSPICE does, while maintaining comparable accuracy. The proposed modeling approaches are theoretically proved to preserve passivity. $\mathrm{Nu}-$ merical experiments on linear network show that the fifth-order DQM-based modeling technique generates solutions at least three times faster than HSPICE.

\section{ACKNOWLEDGMENT}

The authors would like to thank E. S. Kuh and Q. Yu of the University of California at Berkeley for pointing out that our previous work on interconnect modeling by DQM may not guarantee passivity. The fifth-order DQM interconnect modeling has been developed by us to guarantee passivity while retaining the same accuracy.

\section{REFERENCES}

[1] A. R. Djordjevic, T. K. Sarkar, and R. F. Harrington, "Analysis of lossy transmission lines with arbitrary nonlinear terminal networks," IEEE Trans. Microwave Theory Tech., vol. 34, pp. 660-666, 1986.

[2] L. T. Pillage and R. A. Rohrer, "Asymptotic waveform evaluation for timing analysis," IEEE Trans. Computer-Aided Design, vol. 9, pp. 352-377, Apr. 1990.

[3] T. K. Tang and M. S. Nakhla, "Analysis of high-speed VLSI interconnect using the symptotic waveform evaluation technique," IEEE Trans. Computer-Aided Design, vol. 39, pp. 341-352, Mar. 1992.

[4] E. Chiprout and M. S. Nakhla, "Analysis of interconnect networks using complex frequency hopping," IEEE Trans. Computer-Aided Design, vol. 14, pp. 186-200, Feb. 1995.

[5] R. Achar, M. S. Nakhla, and Q.-J. Zhang, "Full-wave analysis of highspeed interconnects using complex frequency hopping," IEEE Trans. Computer-Aided Design, vol. 17, pp. 997-1015, Oct. 1998.

[6] A. B. Khang and S. Muddu, "Two-pole analysis of interconnection trees," Proc. IEEE MCMC Conf., pp. 105-110, 1995.
[7] Q. Yu, J. M. L. Wang, and E. S. Kuh, "Passive multipoint moment matching model-order reduction algorithm on multiport distributed interconnect networks," IEEE Trans. Circuits and Systems-I, vol. 46, pp. 140-160, Jan. 1999.

[8] P. Feldman and R. W. Freund, "Efficient linear circuit analysis by pade approximation via the Lanczos process," IEEE Trans. Computer-Aided Design, vol. 14, pp. 639-649, May 1995.

[9] — - "Reduced-order modeling of large linear subcircuits via a block Lanczos algorithm," Proc. 32nd ACM/IEEE Design Automation Conf., pp. 376-380, 1995.

[10] K. J. Kerns and A. T. Yang, "Stable and efficient reduction of large, multiport RC networks by pole analysis via congruence transformations," IEEE Trans. Computer-Aided Design, vol. 16, pp. 734-744, July 1997.

[11] A. Odabasioglu, M. Celik, and L. T. Pileggi, "PRIMA: Passive reducedorder interconnect macromodeling algorithm," IEEE Trans ComputerAided Design, vol. 17, pp. 645-653, Aug. 1998.

[12] A. R. Djordjevic, T. K. Sarkar, and R. F. Harrington, "Time-domain response of multiconductor transmission lines," Proc. IEEE, vol. 75, pp. 743-764, 1987.

[13] A. C. Cangellaris, M. Celik, S. Pasha, and L. Zhao, "Electromagnetic model order reduction for system-level modeling," IEEE Trans. Microwave Theory Tech., vol. 47, pp. 840-849, June 1999.

[14] B. Fornberg, A Practical Guide to Pseudospectral Methods: Cambridge Univ. Press, Cambridge, U.K., 1996.

[15] M. Celik and A. C. Cangellaris, "Simulation of multiconductor transmission lines using Krylov subspace order-reduction techniques," IEEE Trans. Computer-Aided Design, vol. 16, pp. 485-496, May 1997.

[16] R. Bellman, B. G. Kashef, and J. Casti, "Differential quadrature: A technique for the rapid solution of nonlinear partial differential equations," J. Comp. Phys., vol. 10, no. 1, pp. 40-52, 1972.

[17] Q. Xu and P. Mazumder, "Accurate modeling of lossy nonuniform transmission lines by using differential quadrature methods," IEEE Trans. Microwave Theory Tech., vol. 50, pp. 2233-2246, Oct. 2002.

[18] C. Shu, "Fourier expansion-based differential quadrature and its application to Helmholtz eigenvalue problems," Commun. Numer. Method Eng., vol. 13, 1997.

[19] T. Dhaene and D. D. Zutter, "Selection of lumped element models for coupled lossy transmission lines," IEEE Trans. Computer-Aided Design, vol. 11, pp. 805-815, July 1992.

[20] C. W. Ho, A. E. Ruehli, and P. A. Brennan, "The modified nodal approach to network analysis," IEEE Trans. Circuits Syst., vol. 22, pp. 504-509, June 1975.

[21] R. W. Newcomb, Linear Multiport Synthesis. New York: McGrawHill, 1966.

[22] K. J. Kerns and A. T. Yang, "Preservation of passivity during $R L C$ network reduction via split congruence transformations," IEEE Trans. Computer-Aided Design, vol. 17, pp. 582-591, July 1998.

[23] HSPICE User's Manual, Meta-Software, Inc., 1996.

[24] R. Achar and M. S. Nakhla, "Simulation of high-speed interconnects," in Proc. IEEE, vol. 89, 2001, pp. 693-728.

[25] Q. Xu, Z. F. Li, P. Mazumder, and J. F. Mao, "Time-domain modeling of high-speed interconnects by modified method of characteristics," IEEE Trans. Microwave Theory Tech., vol. 48, pp. 323-327, Feb. 2000.

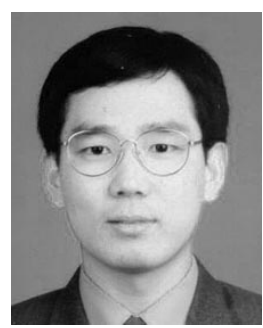

Qinwei Xu (S'02) was born in Shandong, China. He received the B.S. and M.S. degrees in electrical engineering from Shandong University, Shandong, China, in 1991 and 1994, respectively, and is currently working toward the Ph.D. degree in computer science and engineering at the University of Michigan, Ann Arbor.

His research interests include interconnect modeling, circuit simulation, VLSI physical design, and numerical technique in computational electromagnetics. 


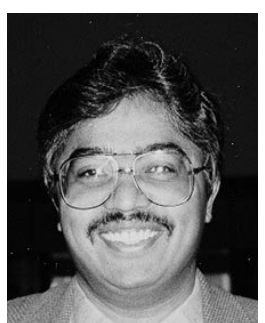

Pinaki Mazumder (S'84-M'88-SM'94-F'99) received the B.S.E.E. degree from the Indian Institute of Science, Bangalore, India, in 1976, the M.Sc. degree in computer science from the University of Alberta, Edmonton, AB, Canada, in 1985, and the Ph.D. degree in electrical and computer engineering from the University of Illinois at Urbana-Champaign, in 1987.

He is currently with the Department of Electrical Engineering and Computer Science, The University of Michigan at Ann Arbor. Prior to this, he spent two years as a Research Assistant with the Coordinated Science Laboratory, University of Illinois at Urbana-Champaign and over six years with Bhara Electronics, Ltd., (a collaborator of RCA), Bangalore, India, where he developed several types of analog and digital integrated circuits for consumer electronics products. During summer of 1985 and 1986, he was a Member of the Technical Staff of the AT\&T Bell Laboratories, Indian Hill. IL. From 1996 to 1997, while on sabbatical, he was a Visiting Faculty Member at Stanford University, Stanford, CA, University of California at Berkeley, and Nippon Telephone and Telegraph, Atsugi-shi, Japan. His research interests include VLSI testing, physical design automation, and ultrafast circuit design. He has lead his research group efforts in VLSI testing and built-in self-repair techniques and has developed silicon compilers for RAM, ROM, and programmable logic array (PLA) with built-in self-repairable capabilities. He has also done extensive work in the area of VLSI physical design. He developed a suite of distributed place-and-route tools for VLSI and field-programmable gate-array (FPGA) chips. For six years, he has been an Integrated Circuit Designer for semiconductor companies. He currently leads ultrafast circuit design activities for nano and quantum electronic devices. He has successfully developed computer-aided design (CAD) tools for high-performance VLSI circuit simulation (NDR SPICE) and numerous circuit topologies for quantum MOS and other quantum-well devices. Several U.S. and Japanese semiconductor companies, including Texas Instruments Incorporated, Hughes Research Laboratory, Lockheed-Martin, NTT, and NEC, have collaborated with him on this research. He has authored or coauthored over 190 papers on these topics in archival journals and proceedings of international conferences. He coauthored Testing and Testable Design of High-Density Random-Access Memories (Boston, MA: Kluwer, 1996), Semiconductor Memories: Testing and Reliability (Los Alamitos, CA: IEEE Comput. Soc., 1998), and Genetic Algorithms for VLSI Design, Layout, and Test Automation (Englewood Cliffs, NJ: Prentice-Hall, 1998). He has two international patents pending. He was Guest Editor of the June 1994 Special Issue on Memory Testing and Reliability, Journal of Electronic Testing Theory and Applications.

Dr. Mazumder is a Member of Sigma Xi, Phi Kappa Phi, and the Association for Computing Machinery (ACM) Special Interest Group on Design Automation (SIGDA). He was a recipient of Digital's Incentives for Excellence Award, the BF Goodrich National Collegiate Invention Award, the National Science Foundation Research Initiation Award, and Bell Northern Research Laboratory Faculty Award. He was Guest Editor of the March 1993 Special Issue on Multimegabit Memory Testing of the IEEE DESIGN AND TEST OF COMPUTERS. He was the Guest Editor of two special issues on Emerging Nanoelectronic Technologies and Their Applications of the IEEE TRANSACTIONS ON VERY LARGE SCALE INTEGRATION SYSTEMS in 1997, and for the PROCEEDINGS OF THE IEEE in 1998. $\mathrm{He}$ is on the Editorial Board of the PROCEEDINGS OF THE IEEE and is an associate editor of the IEEE TRANSACTIONS ON VERY LARGE SCALE INTEGRATION SYSTEMS 